\title{
ARTICLE
}

Received 2 Apr 2013 | Accepted 1 Oct 2013 | Published 25 Oct 2013

DOl: $10.1038 /$ ncomms3691

OPEN

\section{Attosecond nonlinear optics using gigawatt-scale isolated attosecond pulses}

Eiji J. Takahashi ${ }^{1}$, Pengfei Lan ${ }^{1, \dagger}$, Oliver D. Mücke ${ }^{2,3}$, Yasuo Nabekawa ${ }^{1}$ \& Katsumi Midorikawa ${ }^{1}$

High-energy isolated attosecond pulses required for the most intriguing nonlinear attosecond experiments as well as for attosecond-pump/attosecond-probe spectroscopy are still lacking at present. Here we propose and demonstrate a robust generation method of intense isolated attosecond pulses, which enable us to perform a nonlinear attosecond optics experiment. By combining a two-colour field synthesis and an energy-scaling method of high-order harmonic generation, the maximum pulse energy of the isolated attosecond pulse reaches as high as $1.3 \mu \mathrm{J}$. The generated pulse with a duration of 500 as, as characterized by a nonlinear autocorrelation measurement, is the shortest and highest-energy pulse ever with the ability to induce nonlinear phenomena. The peak power of our tabletop light source reaches $2.6 \mathrm{GW}$, which even surpasses that of an extreme-ultraviolet free-electron laser.

\footnotetext{
${ }^{1}$ Extreme Photonics Research Group, RIKEN Center for Advanced Photonics, 2-1 Hirosawa, Wako-shi 351-0198, Japan. ${ }^{2}$ Ultrafast Optics and X-Rays Division Center for Free-Electron Laser Science, Deutsches Elektronen-Synchrotron DESY, Notkestrasse 85, 22607 Hamburg, Germany. ${ }^{3}$ The Hamburg Center for Ultrafast Imaging, Luruper Chaussee 149, 22761 Hamburg, Germany. † Present address: School of Physics, Huazhong University of Science and Technology, Wuhan 430074, China. Correspondence and requests for materials should be addressed to E.J.T. (email: ejtak@riken.jp).
} 
A ttosecond science has emerged as an important research area of ultrafast phenomena within the past decade ${ }^{1}$. Owing to its numerous successes, attosecond science has created important new knowledge (see review ref. 2) of the fundamental science of the interaction between electrons and photons. Recently, some key research topics have been identified, which are expected to make major breakthroughs for the next attosecond frontiers, such as the $\mathrm{MHz}$ high-repetition high-order harmonic $(\mathrm{HH})$ frequency combs for the joint frontier of precision spectroscopy and ultrafast science, attosecond-pump/ attosecond-probe experiments for observing and controlling electronic processes in atomic and molecular physics, and nonlinear science occurring on the attosecond time scale.

To seriously tackle the above interesting research topics, one of the most important issues is the development of high-power isolated attosecond pulses (IAPs) and/or attosecond pulse trains. IAPs and attosecond pulse trains are produced using $\mathrm{HH}$ generation $(\mathrm{HHG})^{3,4}$ in gases. To date, high-power attosecond pulse trains have successfully been generated owing to research on $\mathrm{HH}$ energy scaling using a loose-focusing geometry ${ }^{5}$. Attosecond pulse trains with sufficiently high energy in the microjoule range with a high conversion efficiency on the $10^{-4}$ level were generated ${ }^{6}$, which are intense enough for implementing some applications, such as nonlinear attosecond optics ${ }^{7,8}$ without the assistance of laser pulses, single-shot femtosecond holography $^{9}$ and an external injector for a seeded ${ }^{10}$ freeelectron laser (FEL) in the extreme-ultraviolet (XUV) region. In contrast, the output energy of IAPs is still not sufficient for various applications, although the shortest pulse duration achieved is sub-100 as in ref. 11 . The widespread application of IAPs has been limited because of the low photon flux and the complexity of the laser systems required to produce IAPs (see review in ref. 12). At present, the highest IAP energy is less than a few tens of nanojoules ${ }^{13}$ at a $27-\mathrm{eV}$ photon energy. The highest conversion efficiency demonstrated is only $10^{-5}$ to $10^{-6}$, even if $\mathrm{Xe}$ gas is used. Consequently, the production, characterization and application of high-energy IAPs are still under active investigation.

Here we report a harmonic generation method suitable for nonlinear pump/probe experiments that demonstrates the capability of time-resolving attosecond dynamical processes with IAPs. Our generation scheme is robust and straightforward for scaling up the $\mathrm{HH}$ energy, which is based on an infrared twocolour (TC) laser field synthesis ${ }^{14}$ and the energy-scaling method $^{5}$. By carefully designing the HHG configuration, we can obtain IAPs with a record energy of up to $1.3 \mu \mathrm{J}$ at $\sim 30 \mathrm{eV}$, thus demonstrating a $>100$-fold energy enhancement compared with previous reports ${ }^{13}$. The conversion efficiency achieved is improved to $1.1 \times 10^{-4}$, owing to favourable phase-matching technique. Even though our TC method leads to weak multiple burst emissions ${ }^{14}$ occurring for specific combinations of the carrier-envelope phase (CEP) and relative phase, we nevertheless can observe the nonlinear signal corresponding to IAPs, because the nonlinear process automatically selects predominantly the contributions from the most intense IAPs generated for certain favourable phase combinations. In fact, we present the direct temporal characterization of an IAP by a second-order autocorrelation (AC) measurement using nonlinear phenomena in $\mathrm{N}_{2}$ molecules. The AC measurement is one of the simplest applications of a nonlinear pump/probe experiment. The measured AC traces indicate not only the temporal duration of the interacting IAPs but also provide evidence for the nonlinear interaction with the target medium. From the 500-as pulse duration determined by the AC measurement, the IAP's peak power can be evaluated to be $2.6 \mathrm{GW}$ with weak satellite pulses at the generation point. Furthermore, in our TC-HHG experiment, we create a mid-plateau region exhibiting a quasi-continuous spectrum with an energy of $10 \mu \mathrm{J}$. From the AC measurement of this quasi-continuous spectrum at the mid-plateau, we evaluate that a quasi-IAP with pulse duration of 375 as with $\sim 0.27$ intensity satellite pulses at $\pm 6.7 \mathrm{fs}$ dominated the nonlinear interaction with the $\mathrm{N}_{2}$ target.

\section{Results}

Harmonic spectra and output energy. We strictly considered and designed the configuration of a thoroughly engineered generation scheme (see Methods section) for scaling up the $\mathrm{HH}$ output energy. Figure 1 shows typical single-shot $\mathrm{HH}$ spectra of $\mathrm{Xe}$ gas as function of photon energy. The focused intensity in the TC case $\left(I_{\mathrm{TC}}\right)$ was $\sim 5 \times 10^{13} \mathrm{~W} \mathrm{~cm}^{-2}$ with an intensity ratio of $\xi \sim 0.15$. The $\mathrm{HH}$ yield is optimized by adjusting the focusing point with respect to the gas cell and by varying the gas pressure. As the difference between the dispersions for the 800- and 1,300$\mathrm{nm}$ pulses in $\mathrm{Xe}$ is negligible, the phase-matching condition can be satisfied by optimizing the gas pressure. The $\mathrm{HH}$ yield gradually increases as the Xe gas pressure increases, and exhibits a quadratic dependence on pressure. This means the conversion efficiency and the photon flux of HHG depend on the Xe gas pressure. The cutoff intensity of TC-HHG is optimized at a gas pressure of $\sim 0.8$ Torr, which is in good agreement with the optimum gas pressure predicted by the phase-matching theory ${ }^{15}$ under these experimental conditions.

When the one-colour (OC) field is focused to an intensity of $7 \times 10^{13} \mathrm{~W} \mathrm{~cm}^{-2}$, the $\mathrm{HH}$ spectrum has a discrete structure (blue curve). In the TC scheme (red curve), a quasi-continuous $\mathrm{HH}$ spectrum appears in the lower-order region, whereas the quasicontinuous $\mathrm{HH}$ disappears at harmonic orders higher than the 17 th. We can clearly see a smooth continuum $\mathrm{HH}$ spectrum around the $\mathrm{HH}$ cutoff region. Note that the intensity of the $\mathrm{TC}-\mathrm{HH}$ is comparable to that of the OC-HH. From the wavelength-scaling law of the TC-HHG (see Fig. 8) under the $\xi=0.15$ condition, the conversion efficiency decreases only by a factor of $(1,300 / 800)^{-0.7} \sim 0.71$ compared with the OC-HHG case. The maximum $\mathrm{HH}$ photon energy is slightly extended to $35 \mathrm{eV}$ even though the focused intensity is lower than for the OC-HHG. This photon energy extension is explained by the cutoff formula for TC-HHG ${ }^{14}$.

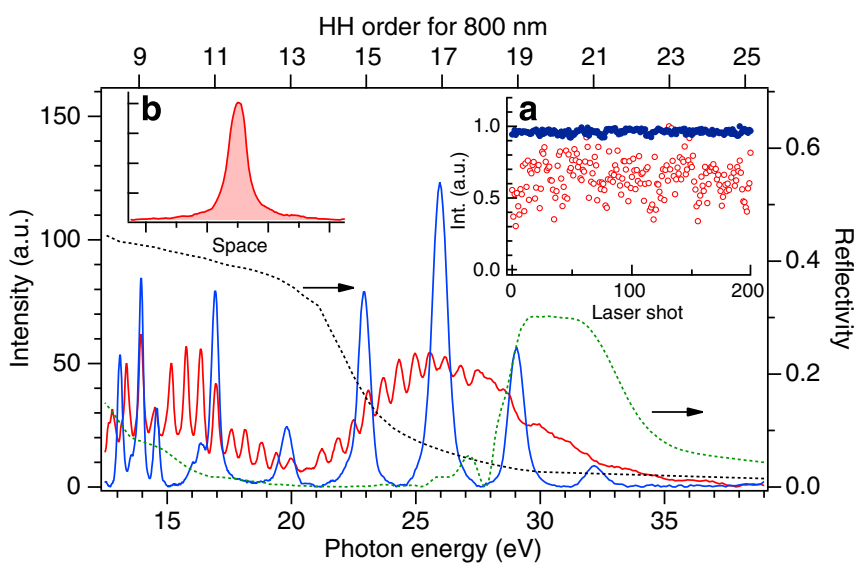

Figure 1 | Experimentally obtained single-shot $\mathrm{HH}$ spectra from Xe. Blue curve: OC laser field $(800 \mathrm{~nm}: 11 \mathrm{~mJ})$; red curve: TC laser field $(800 \mathrm{~nm}$ : $9 \mathrm{~mJ}+1,300 \mathrm{~nm}: 2.5 \mathrm{~mJ}$ ). Green dotted curve: reflectivity of the $\mathrm{Sc} / \mathrm{Si}$ multilayer mirror; black dotted curve: reflectivity of the $\mathrm{SiC}$ bulk mirror. Inset: (a) measured intensity variation of the $\mathrm{HH}$ cutoff ( $\sim 29 \mathrm{eV}$ : 19th $\mathrm{HH}$ order) over 200 laser shots. (b) The spatial profile of the cutoff harmonics of the TC-HHG. 
The inset (a) of Fig. 1 shows the measured intensity variation of the HH cutoff ( $\sim 29 \mathrm{eV}: 19$ th HH order) over 200 laser shots. The intensity of the OC-HH has a high stability, as shown by the blue points, whereas the intensity of the TC-HH (red points) fluctuates between 1 and 0.3 . As already reported in ref. 14, these variations are due to the intensity changes of the TC field caused by random fluctuations in the CEP value of the non-CEP-stabilized Ti:sapphire laser and slight jitter of the relative phase introduced by the Mach-Zehnder interferometer used for creating the TC fields. According to the discussion of the variations of these two kinds of phase effects (see Discussion section), the $\mathrm{HH}$ yield at the cutoff should be maximized, when the continuous spectrum frequently appears for certain 'good' combinations of the CEP and relative phase, whereas only weak $\mathrm{HH}$ exhibiting the quasi-continuum spectrum is generated for 'bad' phase combinations. We have actually observed this correlation between the $\mathrm{HH}$ yield and the spectral shape in the experiment. Note that harmonic shots with the quasi-continuum spectrum hardly contribute to form the AC trace, because their intensity is quite low.

The output beam divergence of the TC-HH at the cutoff was measured to be $\sim 0.5 \mathrm{mrad}$ full-width half maximum with a Gaussian-like profile (see Fig. $1 \mathrm{~b}$ inset). The almost perfect Gaussian profile of the $\mathrm{HH}$ suggests that there is no density disturbance due to high ionization in the harmonic medium. The pulse energy of the continuous $\mathrm{HH}$ spectrum $(28-35 \mathrm{eV})$ is evaluated to be $1.3 \mu \mathrm{J}$ with a conversion efficiency of $1.1 \times 10^{-4}$ from the input TC energy $(800 \mathrm{~nm}: 9 \mathrm{~mJ}+1,300 \mathrm{~nm}$ : $2.5 \mathrm{~mJ})$. This value is almost 100 -fold higher than the highest pulse energies ever reported before ${ }^{13}$. The generation efficiency from the Ti:sapphire laser is $3.7 \times 10^{-5}$, because $30 \mathrm{~mJ}$ of Ti:sapphire energy was used for creating the TC field. Moreover, the midplateau region $(14-29.5 \mathrm{eV})$ exhibiting a quasi-continuous spectrum in the TC-HHG has an output energy of $\sim 10 \mu \mathrm{J}$.

Nonlinear AC measurement for attosecond pulses. To evaluate the temporal characteristics of the microjoule $\mathrm{HH}$ fields, we utilized an AC method using a spatially split autocorrelator. We employed two concave mirrors $(R=200 \mathrm{~mm})$ for focusing the
$\mathrm{HH}$ : one is a bulk $\mathrm{SiC}$ mirror for the mid-plateau and the other is a $\mathrm{Sc} / \mathrm{Si}$ multilayer mirror for the cutoff. The reflectivity for each concave mirror is shown in Fig. 1. We have adopted the $\mathrm{N}^{+}$ion yield from fragmenting $\mathrm{N}_{2}$ molecules as the indicator of the second-order AC signal (see Methods section). This choice is motivated by the findings of our earlier experiment ${ }^{8}$, in which the $\mathrm{N}^{+}$ion yield was sufficiently high to obtain the AC trace of an attosecond pulse in the relevant $\mathrm{HH}$ photon energy region. The dotted curves in the top panels of Fig. 2a,b are the measured AC traces of the mid-plateau $\mathrm{TC}-\mathrm{HH}$ and $\mathrm{OC}-\mathrm{HH}$ fields, respectively. The signals at each delay time are obtained by accumulating signals for $1 \times 10^{3}$ laser shots. We can see distinct peaks emerge at every half period $(1.33 \mathrm{fs})$ of the Ti:sapphire laser field in the AC trace depicted in the top panel of Fig. $2 b$. This result ensures that our AC measurement can certainly reveal the temporal shape in the attosecond regime. In contrast, the four peaks corresponding to multiple bursts on both side of the main peak ( $0 \mathrm{fs}$ ) are well suppressed in the TC-HH, even though we can observe the side peaks at $\sim \pm 6.7 \mathrm{fs}$ (indicated by arrows in Fig. 2a) with a relative AC amplitude of $\sim 0.45$ compared with the main peak. These two side peaks are consistent with our simulated result (see Discussion section) of the TC-HHG.

To observe the pulse duration more precisely, we increased the time resolution of the AC measurement by reducing the delay step from 148 to 28 as. The bottom panels in Fig. 2 show the measured AC signal for OC-HH (blue points) and TC-HH (red points). In the mid-plateau of $\mathrm{TC}-\mathrm{HH}$, the full-width half maximum of the AC was measured to be $\tau_{\mathrm{AC}}=(530 \pm 75)$ as by fitting the trace with a Gaussian function (not shown). The pulse duration is thus $\tau_{\mathrm{HH}}=\tau_{\mathrm{AC}} / \sqrt{2} \sim 375$ as. On the other hand, the measured AC duration for the OC-HHG case was $\tau_{\mathrm{AC}}=(490 \pm 60)$ as. Thus, the duration of each peak in the attosecond pulse train is evaluated to be $\tau_{\mathrm{HH}} \sim 350$ as, which is slightly shorter than the pulse duration of the TC-HH. The broadening of the AC duration of the $\mathrm{TC}-\mathrm{HH}$ compared with the $\mathrm{OC}-\mathrm{HH}$ case is owing to the fact that the AC trace of the TC-HH originates from the temporal profiles averaged over the random CEP and slightly fluctuating relative phase. In addition, the $\mathrm{HH}$ spectral shapes are different in both cases.

b
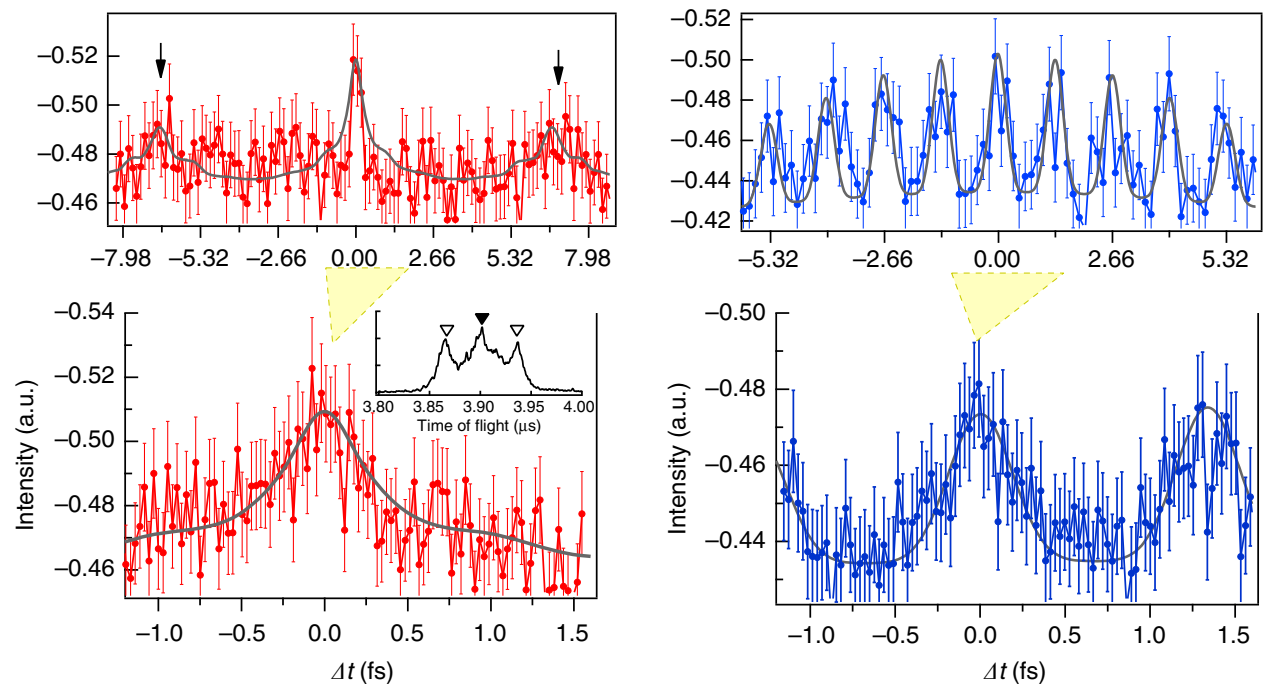

Figure 2 | Measured AC traces from the side peak of $\mathbf{N}^{+}$ion signals. (a) Mid-plateau of TC-HH; (b) mid-plateau of OC-HH. The time resolutions of the top and bottom panels correspond to 148 and 28 as, respectively. The error bars show the s.d. of each data point. The grey solid profiles are the AC traces of the simulated $\mathrm{HH}$ spectrum. Inset of (a): measured TOF spectrum of ions at around $\mathrm{m} / \mathrm{z}=14$. The inverted triangles and black-filled inverted triangle correspond to the $\mathrm{KE}$ of 3 and $\mathrm{O} \mathrm{eV}$, respectively. 
As we successfully obtained the AC trace of the TC-HH in the mid-plateau, we furthermore explored the temporal characterization for the $\mathrm{HH}$ cutoff. In this experiment, we switched the focusing mirror from the $\mathrm{SiC}$ mirror to the $\mathrm{Sc} / \mathrm{Si}$ multilayer mirror. The signal is scanned every 148 as from -8.5 to $8.5 \mathrm{fs}$ with $1 \times 10^{3}$ laser shot accumulation at each delay point. The resultant signal points versus delay are shown in the top panel in Fig. 3. Three distinct signal points around 0 delay seem to be a correlation peak and there are no noticeable side peaks in this figure, although our simulation result (see Discussion section) exhibits side peaks at \pm 6.7 fs with $\sim 0.2$ relative $\mathrm{AC}$ amplitude compared with the maximum peak at 0 delay. To confirm that the signal around 0 delay is certainly the correlation signal and that there are no significant side peaks at $\pm 6.7 \mathrm{fs}$, we performed a high-resolution $\mathrm{AC}$ measurement in the delay scanning ranges around $0 \mathrm{fs}$ and $\pm 6.7 \mathrm{fs}$, as shown in the bottom panels in Fig. 3 . We accumulated $1 \times 10^{3}$ laser shots at each delay point and set the scanning delay step $\Delta t$ to be 28 as. We can demonstrate a clear peak at 0 delay as an $\mathrm{AC}$ in the middle-bottom panel in Fig. 3. The AC duration was evaluated to be $\tau_{\mathrm{AC}}=(700 \pm 90)$ as by fitting the experimental trace to a Gaussian function; hence, the pulse duration is estimated to be $\tau_{\mathrm{HH}} \sim 500$ as. We also find that there are no noticeable side peaks in the measured AC traces in the leftbottom and right-bottom panels in Fig. 3. This is owing to the fact that the background noise amplitude (see Methods section) is comparable to the amplitude of the side peaks expected from our simulation. The noise magnitude against the peak AC amplitude is estimated to be $\sim 0.24$; thus, we cannot clearly find side peaks with amplitudes $<0.24$. In other words, we can safely conclude that the side-peak ratio should be $<0.24$. As discussed below, we can find both pre- and post-pulse in the calculated temporal profile at any $\mathrm{CEP}$ and relative phase. By assuming that the $\mathrm{HH}$ pulse consists of three (pre-, main and post-) pulses and the upper limit of the side peaks in the AC trace should be 0.24 , we can estimate the possible largest peak height ratio of the pre- and post-pulses to be $\sim 0.14$. Further detailed analysis of the pre-/post-pulse issue is presented in the next section with the help of our numerical model of the TC-HH and statistical analysis of the relative phase.

From the measured pulse energy and pulse duration, the peak power of the IAP nonlinearly interacting with the target medium is evaluated to be $2.6 \mathrm{GW}$ with weak satellite pulses (see Discussion section). This ultrahigh power from our tabletop light source even surpasses that from a compact XUV FEL ${ }^{16,17}$. The peak brightness is also estimated to be $\sim 2 \times 10^{30}$ photons per $\mathrm{s}^{-1} \mathrm{~mm}^{-2} \mathrm{mrad}^{-2}$, assuming a $60-\mu \mathrm{m}$ beam diameter at the exit of the gas cell.

\section{Discussion}

Finally, we examine attosecond pulse generation in our TC-HH scheme by numerical simulations. In these simulations, the single-atom response is calculated within the strong-field approximation. The propagation of the TC laser pulse and the high harmonics are computed separately in cylindrical coordinates ${ }^{14,18}$. All the parameters, such as laser intensity, target gas pressure and medium length, are determined by the experimental conditions. Here we assume that the CEP $\phi_{\mathrm{CE}}$ fluctuates randomly during the $\mathrm{AC}$ measurement, whereas the statistical fluctuations of the relative phase $\phi_{0}$ are characterized by the measured histogram of the relative phase in the TC interferometer. To evaluate the relative phase variation with time in the TC interferometer, we injected a continuous-wave (CW) laser beam with a wavelength of $547 \mathrm{~nm}$ into the TC interferometer in this measurement. We recorded spatial interference fringes on the superposed beam profile of the CW laser with a charge-coupled device camera (exposure time: $\sim 10 \mu$ s with $10 \mathrm{~Hz}$ repetition rate, which is synchronized with the pump laser) during $\sim 35 \mathrm{~min}$, which is equivalent to the data acquisition time of one AC trace in our measurements. The phase of the spatial fringes at each recorded profile was converted to the relative delay and relative phase corresponding to the $1,300-\mathrm{nm}$ field. The measured time evolution of the relative phase, $\phi_{0}$, is shown in Fig. $4 \mathrm{a}$. We can then evaluate the s.d. of $\phi_{0}$ fluctuations, $\sigma$, to be $0.23 \pi$ from this measurement, whereas the offset of $\phi_{0}$ is arbitrary. We adjust the offset in Fig. 4a such that the average of $\phi_{0}$ is equal to 0 . To improve the $\mathrm{S} / \mathrm{N}$ ratio of the $\mathrm{AC}$ trace, we overlapped ten $\mathrm{AC}$ traces in Figs 2 and 3. The $\sigma$-value with a typical normal distribution of the ten $\mathrm{AC}$ traces' $\phi_{0}$ fluctuations was almost the same during the experiment, even though the phase profile as a function of time was different.



Figure 3 | Measured AC traces of an IAP obtained from the side peak of $\mathbf{N}^{+}$ion signals. The time resolutions of the top and bottom panels correspond to 148 and 28 as, respectively. The error bars show the s.d. of each data point. The grey solid profiles are AC traces obtained using the simulated HH fields. 
The histogram of our measured relative phase exhibits a typical normal distribution expressed by $\sim \exp \left[-\left(\phi_{0}-\left\langle\phi_{0}\right\rangle\right)^{2} /\left(2 \sigma^{2}\right)\right]$ with a s.d., $\sigma$, of $0.23 \pi$ (see Fig. $4 \mathrm{~b}$ ). The mean value of $\phi_{0}$, denoted as $\left\langle\phi_{0}\right\rangle$ in this equation, cannot be determined by our jitter measurement. Nevertheless, we set $\left\langle\phi_{0}\right\rangle$ to be $0.14 \pi \mathrm{rad}$ in our averaging procedure in the calculations for reasons discussed below. Using the parameters mentioned above, we first calculate the temporal intensity profile for each $\phi_{0}$ (calculation step: $100 \mathrm{mrad})$ value, varying $\phi_{\mathrm{CE}}$ in the range from $-\pi / 2$ to $\pi / 2$; later, we average the calculated $\mathrm{HH}$ intensity profile. We call the temporal profile of this averaged field the 'CEP-averaged temporal profile' at each $\phi_{0}$ (see Fig. 5). Note that we adjust the offset of the time axis of each temporal profile of the $\mathrm{HH}$ field so as to locate the highest peak at the time origin before CEP averaging. This adjustment is consistent with the temporal profile to be measured with the spatially split AC technique, because the delay time $(\Delta t)$ at the highest $\mathrm{AC}$ signal does not indicate the absolute time of the $\mathrm{HH}$ emission with respect to the driving TC-HH field, but coincides with the zero delay time between two replicas of the measured pulse. In an actual setup (see Fig. 9), we produce two time-delayed replica pulses from one $\mathrm{HH}$ pulse using two silicon beam splitters placed at Brewster's angle for the driving field ${ }^{19}$.
After performing the CEP average, we superpose the 'CEPaveraged temporal profiles' at each $\phi_{0}$ (see Fig. 5) with the abovementioned weighting factor of the normal distribution of $\phi_{0}$ (see solid black curve in Fig. 4b). We have numerically searched for the mean value, $\left\langle\phi_{0}\right\rangle$, in the normal distribution so as to minimize the side peaks of the superposed temporal profile, and then found $\left\langle\phi_{0}\right\rangle$ to be $0.14 \pi \mathrm{rad}$ as mentioned above. This numerical procedure is compatible with our experimental approach of adjusting the relative phase in the TC interferometer such that the TC-HH exhibits the continuous spectrum most frequently. Thus, it is justified to set $\left\langle\phi_{0}\right\rangle$ to be $0.14 \pi \mathrm{rad}$ in the normal distribution. The resulting $\phi_{0}$-weighted superposition of the 'CEP-averaged temporal profile', which we call the 'averaged temporal profile', in the mid-plateau region is shown as red-filled curve in Fig. $6 \mathrm{a}$ and that in the cutoff region is shown as greenfilled curve in Fig. 6b. The averaged temporal profile includes both the effect of random fluctuation of $\phi_{\mathrm{CE}}$ and the measured relative phase jitter of $\phi_{0}$. The overall selected bandwidths of the mid-plateau and cutoff harmonics are $13-29 \mathrm{eV}$ and $27-36 \mathrm{eV}$, which are determined by the reflectivity of the $\mathrm{SiC}$ and $\mathrm{Sc} / \mathrm{Si}$ mirrors, respectively. Note that $13-23 \mathrm{eV} \mathrm{HH}$ effectively contribute to creating the temporal profile in the mid-plateau
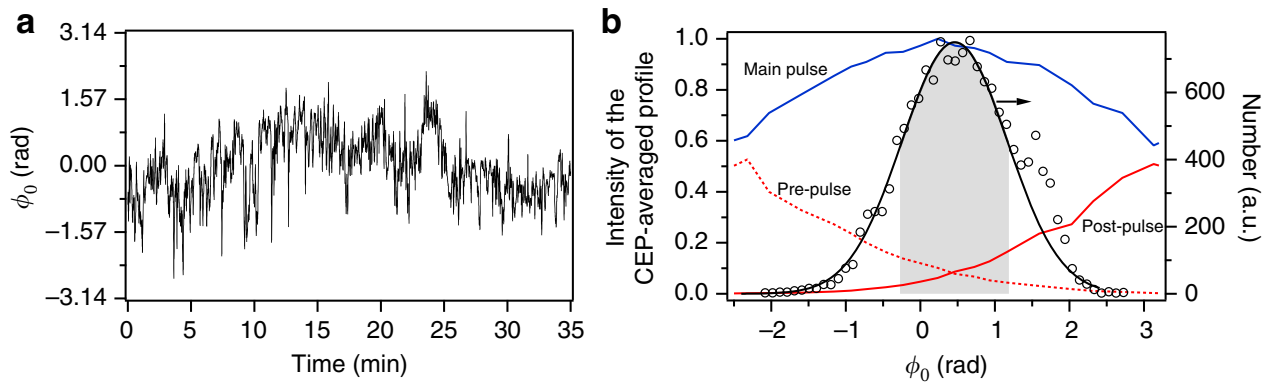

Figure 4 | Evaluation of the relative phase. (a) Measured relative phase versus time. (b) A histogram obtained from a with a normal distribution fit (black solid curve). The intensity ratio of the CEP-averaged profile of the main (blue solid curve), pre- (red dotted curve) and post-pulse (red solid curve). The grey-filled area corresponds to the $\pm \sigma$ area.
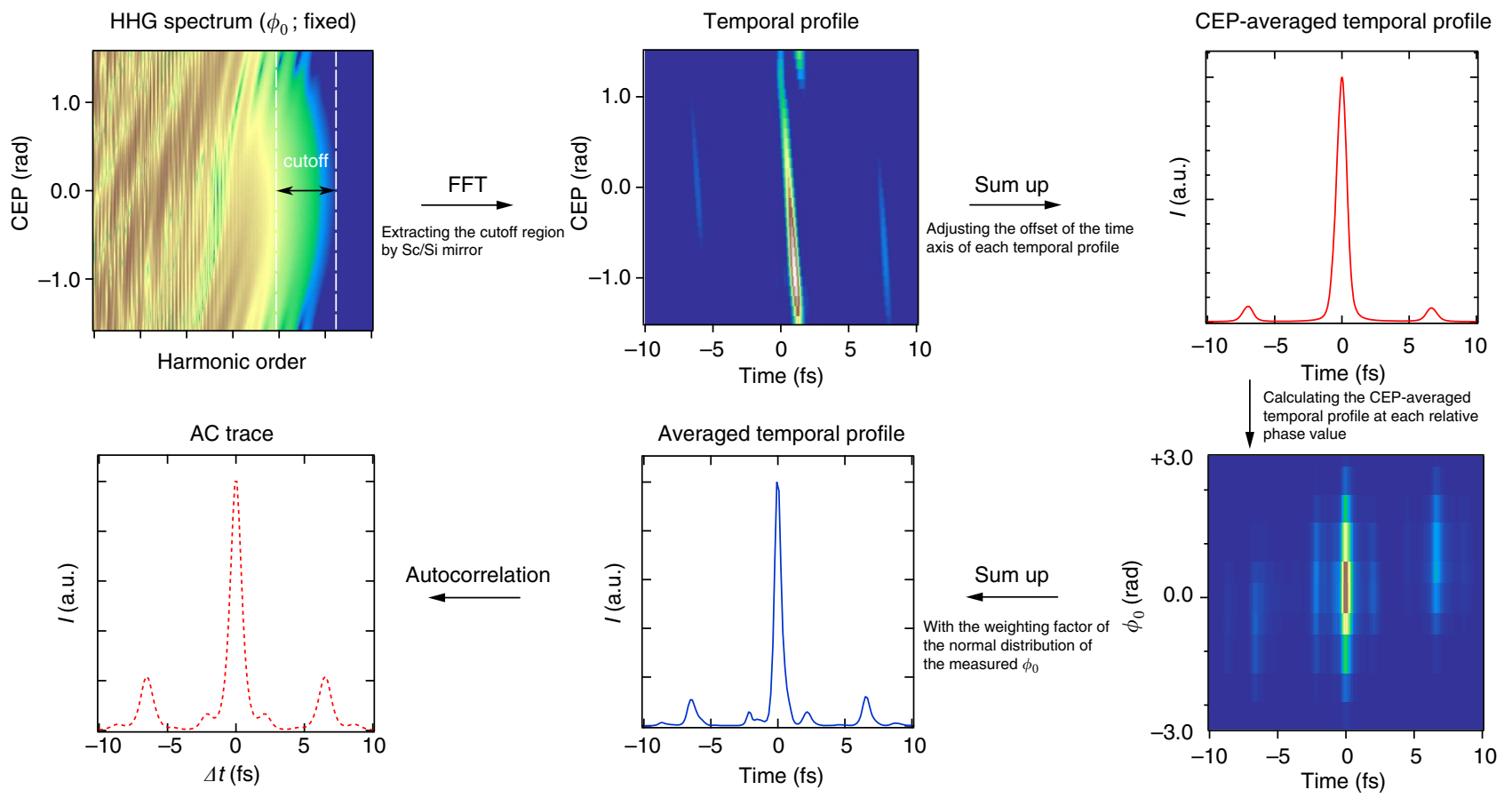

Figure 5 | Calculation procedure. Calculation procedure for evaluating the temporal profile of HHs. 
TC-HH profile due to the reflectivity edge of the $\mathrm{SiC}$ mirror. We notice that two satellite pulses appear at $\pm 6.7 \mathrm{fs}$ in both TC-HH profiles. The relative intensity of the higher satellite pulse compared with the main pulse for the mid-plateau TC-HH is 0.27 . In contrast, the relative intensity of the cutoff TC-HH is considerably decreased to 0.1 . Note that this side-peak intensity can be decreased by extracting slightly higher cutoff harmonic orders $(>29 \mathrm{eV})$. We also observe a background pedestal under the main pulse in both $\mathrm{TC}-\mathrm{HH}$ profiles. This small pedestal originates from weak multiburst emission around the centre of the TC electric field at the 'wrong' combinations of CEP and relative phase. The intensity of this pedestal is very low, because the $\mathrm{HH}$ yield is also low. Even though the temporal profile has side peaks $(\sim 0.27$ relative intensity) at $\pm 6.7 \mathrm{fs}$, the mid-plateau of TC-HH results in an IAP profile having a 390 -as temporal duration for the central pulse. When we extract the cutoff harmonics, the expected temporal duration is 525 as with weak satellite pulses. We also calculated the AC trace by averaging over the CEP and relative phase according to the procedure shown in Fig. 5, the simulated results are shown by dashed curves in Fig. 6 and solid curves in Figs 2 and 3. As shown in these figures, the calculated AC traces are in good agreement with the experimental data. We can clearly find a significant reduction of the pulses forming an attosecond pulse train with a period of $1.33 \mathrm{fs}$ in both $\mathrm{TC}-\mathrm{HH}$ fields by comparing these profiles with that of the $\mathrm{OC}-\mathrm{HH}$ in the mid-plateau region. Thus, we can conclude that the intensity of multiple burst emissions in our TC-HHG can be significantly reduced compared with another gating method ${ }^{20,21}$. In fact, the previously reported AC profile ${ }^{21}$ exhibits a duration in the femtosecond region when a non-CEPstabilized multicycle driving laser was utilized for generating IAPs. We notice that the intensity ratio between the main ( $0 \mathrm{fs})$ and the satellite pulses ( $\pm 6.7 \mathrm{fs}$ ) of the AC traces is much higher (approximately twice) than that of the temporal profiles shown in Fig. 6, respectively. As the temporal profile of the TC-HH cutoff is composed by three pulses, the simulated AC intensity ratio of the side peak is $\sim 0.2$ compared with the main pulse, even though the temporal intensity ratio of the side peak is $\sim 0.1$.

In addition, we discuss in detail how these pre- and postpulses at $\pm 6.7 \mathrm{fs}$ affect the measured temporal profiles with the statistical fluctuations of these phases. Here we do not consider the background pedestal under the central main pulse (in the region within $\pm 2.7 \mathrm{fs}$ ) originating from weak multiple burst emissions. In Fig. $4 b$, the peak intensity of the main pulse, the pre-pulse and the post-pulse are plotted versus $\phi_{0}$ as blue solid, red dotted and red solid curves, respectively. We can see that the

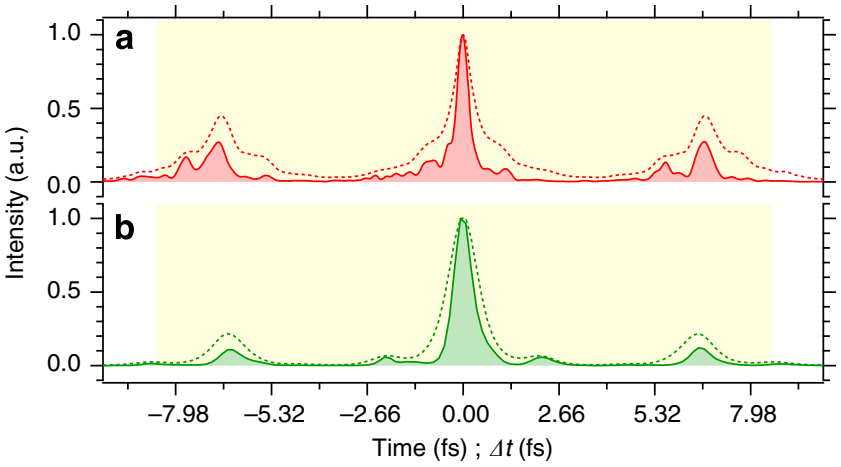

Figure 6 | Averaged temporal profiles and their AC traces in TC-HHG. (a) Mid-plateau of $\mathrm{TC}-\mathrm{HH}$; (b) cutoff of $\mathrm{TC}-\mathrm{HH}$. The filled curves and dashed curves exhibit the averaged temporal profiles and their AC traces, respectively. The yellow highlighted region shows the time interval measured in the experiment. peak intensity of the main pulse reaches its maximum height at a relative phase of $0.14 \pi$, and the pre- and post-pulses are most effectively suppressed at this symmetrical point with peak heights of both 0.08 . We find that the highest peak of the pre- and postpulses in the $\pm \sigma$ region around $\left\langle\phi_{0}\right\rangle$ is lower than $1 / e^{2} \sim 0.14$, whereas the area of the normal distribution in our $\phi_{0}$ range, which accounts for $68.3 \%$ of all events, is depicted as grey-filled area in Fig. 4b. In the second-order AC experiment, the AC intensity of main pulse $\left(I_{\mathrm{AC}-\text { main }}\right)$ and the side pulse $\left(I_{\mathrm{AC}-\text { side }}\right)$ are given by the square of the main pulse of the CEP-averaged temporal profile $\left(I_{\text {main }}^{2}\right)$ and $I_{\text {main }}\left(I_{\text {pre }}+I_{\text {post }}\right)$, respectively. Our simulation shows that the event rate of the $\pm \sigma$ region, which has weak pre/post pulse in Fig. $4 \mathrm{~b}$, contributes $\sim 85 \%$ to the whole AC trace. From this evaluation, we can conclude that the AC signal at the foot of our relative phase distribution (outside the $\pm \sigma$ region) is negligible for composing the $\mathrm{AC}$ trace. In other words, the two-photon nonlinear process automatically picks up the most intense IAP part for specific combinations of the CEP and relative phase.

As our method has the advantage that the $\mathrm{HH}$ output yield can be linearly scaled up by increasing the $\mathrm{HH}$ emission volume, we now discuss a scaled-up configuration in the soft-X-ray region to obtain even shorter IAP durations. In the soft-X-ray region at around $100 \mathrm{eV}$, we have already demonstrated an $\sim 30 \mathrm{~nJ}$ energy per harmonic order from $\mathrm{OC}-\mathrm{HH}$ in $\mathrm{Ne}$ gas $^{22}$. By straightforwardly upgrading the TC-HHG to a main pump energy of $50 \mathrm{~mJ}$ (with $\xi=0.15$ ) and adopting a focusing length of $5 \mathrm{~m}$ with a $5-\mathrm{cm}$-long $\mathrm{Ne}$ medium, we can expect to achieve $\mathrm{HH}$ pulse energies $>0.1 \mu \mathrm{J}$ from 95 to $110 \mathrm{eV}$, which is almost 1,000 -fold higher than the energies previously reported ${ }^{11}$. Our simulations indicate that IAPs can be generated with sub-300 as duration. In addition, a straightforward upscaling of the IAP power to a few tens of gigawatts is feasible by extending the focusing length to $50 \mathrm{~m}$ with a $100-\mathrm{mJ}$ main pump energy (with $\xi=0.15$ ), while maintaining a pulse duration of 500 as at $\sim 30 \mathrm{eV}$.

Since the first demonstration of an IAP was reported in 2001 (ref. 1), continuous effort to increase the IAP energy ${ }^{12}$ has been exerted by many research groups around the world over the past decade, as illustrated in Fig. 7. Unfortunately, the output energy of IAPs has not exceeded the nanojoule level thus far. Owing to the low photon flux, the temporal characterization of the IAP was

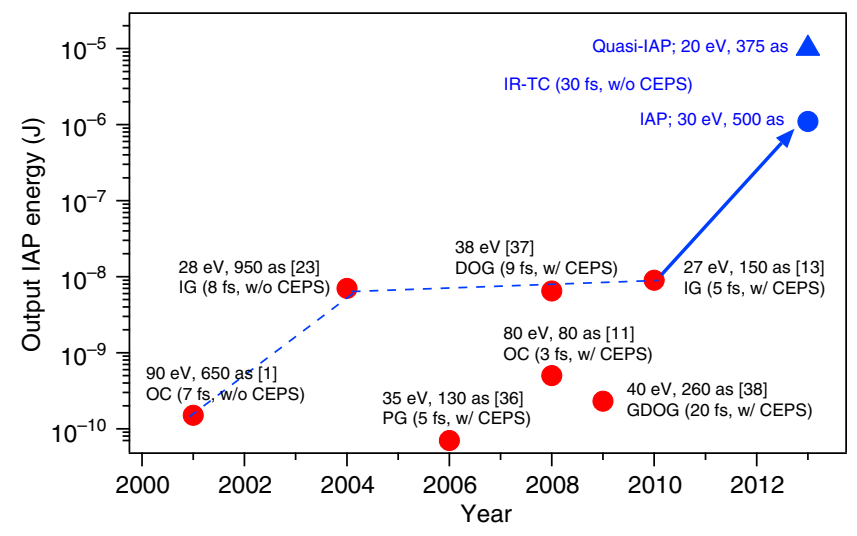

Figure 7 | Progress of the energy of IAPs used in attosecond experiments. Red circles: previous milestone results using various schemes; blue points: our HHG source. IG, ionization gating; PG, polarization gating; DOG, double optical gating; GDOG, generalized DOG. We indicate the required laser pulse duration and CEP stabilization (CEPS) for each generation scheme. Note the dramatic leap in IAP energy, which is used in an attosecond experiment, by two orders of magnitude achieved in this work. 
generally achieved using the attosecond streaking method employing frequency-resolved optical gating for complete reconstruction of attosecond bursts (FROG-CRAB) reconstruction. We have now improved the available IAP energy for nonlinear optics experiments by two orders of magnitude compared with the highest output energy reported previously ${ }^{23}$. Our method features good energy scalability and high conversion efficiency owing to the phase-matching technique. We directly characterized the temporal profile of our IAP by an AC method using the nonlinear interaction of $\mathrm{N}_{2}$. In fact, the $\mathrm{AC}$ traces clearly demonstrate that our $\mathrm{HH}$ source is capable of not only inducing nonlinear phenomena but also having attosecond temporal resolution. This experiment also demonstrates the capability of our technique to observe nonlinear attosecond dynamics in real time with IAPs by time-resolved spectroscopy employing a non-sequential twoXUV-photon process in a molecule. Moreover, our intense IAPs are useful as the external injector for a seeded FEL ${ }^{10}$ in the XUV region. By combining our attosecond technology and seeded FEL technology, we might obtain an altogether novel XUV light source in the near future. In addition, the quasi-IAP from the mid-plateau of TC-HH will be useful for several applications, for example, for the control of electron localization ${ }^{24}$ by attosecond pulse trains, the manipulation of the HHG process ${ }^{25,26}$ and pump-probe experiments involving a few-cycle laser pulse. Now, the IAP energy has reached the microjoule level, which is 10,000 times higher than the energy of the first demonstration. We think

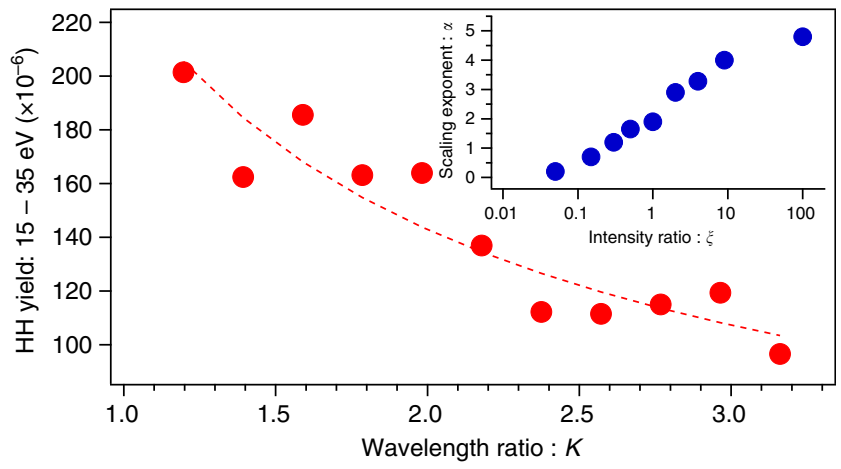

Figure 8 | $\mathrm{HH}$ yield as a function of the wavelength ratio $K=\lambda_{1} / \lambda_{0}$.

The relative intensity ratio $\xi=I_{\mathbb{R}} / I_{800 \mathrm{~nm}}$ is fixed to be 0.15 . Dashed line: $\alpha=0.7$ scaling. Inset: scaling exponent $\alpha$ (from $\mathrm{HH}$ yield $\propto K^{-\alpha}$ ) as a function of $\xi$. that the presented method paves the way for the intense attosecond science frontier, which will certainly bring a great leap forward in research on ultrafast phenomena and nonlinear optics.

\section{Methods}

Generation configuration for intense attosecond pulses. From the point of view of energy scaling for IAPs, the following essential factors have to be considered: (i) scalability of the pump laser energy, (ii) high conversion efficiency of the HHG and (iii) scalability of the generation geometry of HHG. As proposed and demonstrated in ref. 14, our infrared-TC scheme enables us not only to relax the requirements for the pump pulse duration $(\sim 30 \mathrm{fs})$ and the CEP stabilization but also to markedly minimize the ionization of the harmonic medium. This is a major advantage for efficiently generating intense attosecond pulses, because neutral media allow us to use the phase-matching technique. We strictly considered and designed the configuration of our infrared-TC scheme for scaling up the HH output energy. The supplemental infrared wavelength is optimized to $1,300 \mathrm{~nm}$ to effectively suppress satellite pulses on both sides of the central pulse. To extract the HH energy efficiently, the harmonic medium should be extended to satisfy the absorptionlimited condition ${ }^{15}$ under the phase-matching condition. In the infrared-TC scheme, the difference in group delay between the 800-nm $\left(\lambda_{0}\right)$ and 1,300-nm $\left(\lambda_{1}\right)$ pulses even in $\mathrm{Xe}$ gas is evaluated to be just 9 as in the 12-cm-long medium of $\mathrm{Xe}$ (1 Torr). As this amount is negligible for the synthesized TC field, we can perfectly maintain the waveform in the harmonic medium during propagation.

Next, to achieve the highest possible conversion efficiency of HHG in the infrared-TC scheme, we study the effect of $\xi\left(=I_{\mathrm{IR}} / I_{800 \mathrm{~nm}}\right)$ on the conversion efficiency by numerical simulations. The $\mathrm{HH}$ yield generally decreases with increasing driving wavelength, which is proportional to $\propto \lambda^{-\alpha}$ with $5 \leq \alpha \leq 6$ (ref. 27). To investigate the scaling exponent $\alpha$ in the TC-HHG, we computed the $\mathrm{HH}$ yield by solving the time-dependent Schrödinger equation (TDSE), $i \frac{\partial \psi(\mathbf{r}, t)}{\partial t}=$ $\left[-\frac{\nabla^{2}}{2}+V(r)+z E(t)\right] \psi(\mathbf{r}, t)$, where $V(r)$ is the atomic potential and atomic units are used. We assume that the laser pulse is polarized along the $z$ direction. The TDSE is solved in spherical coordinates, so $z=r \cos \theta$. We consider a hydrogen target and $V(r)=-1 / r$. The linearly polarized electric field is expressed by $E_{\mathrm{TC}}=E_{0} \exp \left[-2 \ln 2\left(\frac{t}{\tau_{0}}\right)^{2}\right] \cos \left(\omega_{0} t+\phi_{\mathrm{CE}}\right)+E_{1} \exp \left[-2 \ln 2\left(\frac{t-\delta t_{1}}{\tau_{1}}\right)^{2}\right]$ $\cos \left(\frac{\omega_{0} t}{K}+\phi_{\mathrm{CE}}+\phi_{0}\right)$, where $K$ is the frequency ratio between the main field $\left(\omega_{0}\right)$ and the supplemental infrared field $\left(K=\omega_{0} / \omega_{1}, K>1\right)$. The subscripts 0 and 1 denote the main fundamental laser and supplemental infrared pulse, respectively. $E$ is the electric field; $\tau_{0,1}, \phi_{\mathrm{CE}}$ and $\phi_{0}$ denote the pulse durations, CEP and relative phase, respectively. Note that the jitter in temporal delay $\delta t_{1}$ and in relative phase $\phi_{0}$ introduced by path-length fluctuations of the interferometer are connected via $\delta t_{1}=K \phi_{0} / \omega_{0}$. Here the pulse durations of the fundamental and infrared pulses are both $30 \mathrm{fs}$, and the total peak intensity is kept constant at $I_{\mathrm{TC}}=6 \times 10^{13} \mathrm{~W} \mathrm{~cm}^{-2}$. To determine $\alpha$ for each $\xi$, the central wavelength of the main pulse is kept at $800 \mathrm{~nm}$ and that of the infrared pulse is varied from $900 \mathrm{~nm}$ to $2,500 \mathrm{~nm}$. $\xi$ is varied from 0 to $\infty$, the two limits corresponding to OC-HH with $\lambda_{0}$ and OC-HH with $\lambda_{1}$, respectively. The TDSE is solved using the method described in ref. 28. The results shown in the inset of Fig. 8 indicate that the scaling exponent $\alpha$ rapidly increases when increasing the relative intensity $\xi$ and finally approaches $\sim 5.5$. A high $\xi$ is disadvantageous for the conversion efficiency, even if a good suppression of the shoulder pulses on both sides of the central pulse as well as an extension of the HH cutoff can be obtained. We investigated an upper limit of $\xi$, for which both a relatively high conversion efficiency and a good

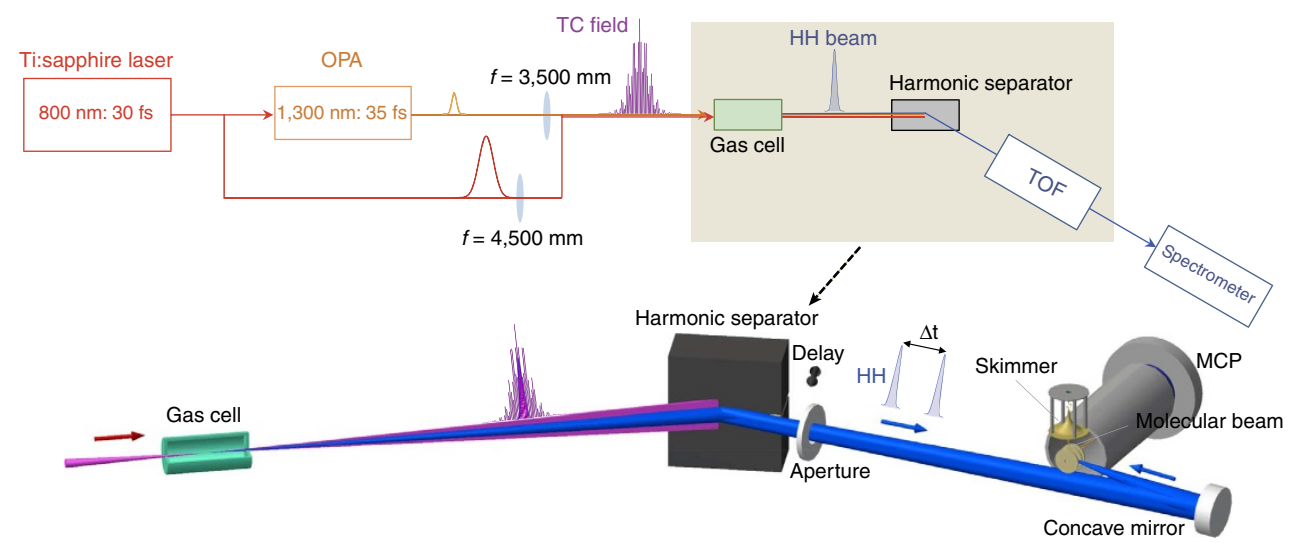

Figure 9 | Schematic figure of the HHG setup and the second-order autocorrelator. The AC setup (bottom part) is composed of a pair of Si harmonic separators and controls the delay $(\Delta t)$ between the two replicas of the reflected pulse. The two replica pulses with $\Delta t$ delay time are focused by a concave mirror ( $\mathrm{SiC}$ bulk or Sc/Si multilayer) onto a molecular beam of $\mathrm{N}_{2}$. The fragment ions from $\mathrm{N}_{2}$ molecules, which are introduced through a skimmer from a pulsed gas jet, are detected by a TOF ion mass spectrograph, which is constructed from three electrodes, a flight tube, and a microchannel plate (MCP) 
suppression of shoulder pulses are simultaneously realized, and we decided to use $\xi \sim 0.15$. For $\xi=0.15$, we find that the HH yield obeys a scaling law of $\sim K^{-0.7}$ with the wavelength ratio $K=\lambda_{1} / \lambda_{0}$ (see Fig. 8). Moreover, we emphasize that our infrared-TC method can universally be applied while maintaining the energyscaling strategy of HHG, independent of the harmonic medium.

Experimental setup. $800-\mathrm{nm}$ and infrared pulses are generated starting from a homebuilt TW-Ti:sapphire laser system $(30 \mathrm{fs}, 150 \mathrm{~mJ}, 800 \mathrm{~nm}, 10 \mathrm{~Hz}$; Fig. 9). Infrared pulses with a 40 -fs duration are produced by a white-light-seeded highenergy optical parametric amplifier ${ }^{29}$. To correct for the different focal lengths of the $800-\mathrm{nm}$ and infrared pulses, both pulses were focused using two separate focusing lenses ( $f=4.5 \mathrm{~m}$ for $800 \mathrm{~nm}, f=3.5 \mathrm{~m}$ for infrared). We discuss the stability of the interferometer part to produce the TC field in the Discussion section. The two beams are collinearly combined with a dichroic beam splitter and delivered into the target chamber through a thin $\mathrm{CaF}_{2}$ window. The focused beams pass through the interaction gas cell, which has two pinholes on the entrance and exit surfaces for maintaining the vacuum. The confocal parameters of the two beams are adjusted to be the same, matching the Gouy phase shift through the focus. The Xe target gas was statically filled into the interaction cell of $12 \mathrm{~cm}$ length. The HH signals were observed with a flat-field normal-incidence XUV spectrometer with a microchannel plate. A charge-coupled device camera detected the two-dimensional fluorescence from a phosphor screen placed behind the microchannel plate.

To evaluate the $\mathrm{HH}$ output energy, we first directly measured the total pulse energy of OC-HH from 11th to 21st HH by using an XUV photodiode. The values of spectral sensitivity and quantum efficiency of the XUV photodiode were obtained from ref. 30 and were calibrated with 266-nm Q-switched YAG laser pulses in this experiment. To perfectly block the pump pulse and lower harmonic components $(<11$ th), we inserted a 200 -nm-thick Al filter supported by a meshback in front of the photodiode. Although the filter transmission of $\mathrm{Al}$ can be estimated from the absorption coefficient, the $\mathrm{Al}$ filter transmission degrades with time because of oxidation ${ }^{31}$. Therefore, the filter transmission for individual harmonics was calibrated using an XUV spectrometer. From the HH spectral distribution measured by the XUV spectrometer, we divided the total pulse energy among the six harmonic components. Thus, for OC-HH we obtain the relationship between the $\mathrm{HH}$ spectral intensity and the pulse energy. In our TC-HH experiments, we computed the area under the measured continuum spectrum by integration within a certain energy range, to evaluate the pulse energy in the cutoff region. When we evaluate the conversion efficiency of $\mathrm{HH}$ in the TC-HH case, we used the total pump energy $(800 \mathrm{~nm}+1,300 \mathrm{~nm})$.

Indicator of the $\mathbf{A C}$ measurement. We have adopted the $\mathrm{N}^{+}$ion yield from fragmenting $\mathrm{N}_{2}$ molecules as the indicator of the second-order AC signal. We show a typical time-of-flight (TOF) mass spectrum of ions at a mass number $\mathrm{m} / \mathrm{z}=14$ for irradiation with the mid-plateau TC-HH field in the inset of the bottom panel of Fig. 2a. We can expect that the ion yields in the TOF spectrum contain the $\mathrm{N}^{+}$ fragment ions from the dissociative state of $\mathrm{N}_{2}^{+}$(ref. 32) and the Coulomb explosion of $\mathrm{N}_{2}^{2+}$, in addition to doubly charged nitrogen molecular ions $\mathrm{N}_{2}^{2+}$ (ref. 33) and/or $\mathrm{N}^{+}$ejected through the decomposition pathway of $\mathrm{N}_{2}^{+}$having zero kinetic energy (KE). The AC function was measured by recording the sidepeak yield at $\mathrm{KE} \sim 3 \mathrm{eV}$ indicated by the inverted triangle (left side) as a function of the delay time $\Delta t$. The photon energy required for creating these KE peaks around $3 \mathrm{eV}$ is expected to be $32 \mathrm{eV}$ or higher, as deduced from high-resolution coincidence measurements of electrons and $\mathrm{N}^{+}$ions ${ }^{32}$. From the reflectivity of our $\mathrm{SiC}$ mirror we conclude that in our situation, the dominant contribution to create the side-peak signals at $\mathrm{KE} \sim 3 \mathrm{eV}$ comes from non-sequential two-photon ionization of $\mathrm{HH}$ with energies from 12 to $21 \mathrm{eV}$. When we replaced the focusing mirror from $\mathrm{SiC}$ to $\mathrm{Sc} / \mathrm{Si}$, we observed the other $\mathrm{KE}$ peak of $\mathrm{N}^{+}$at $\sim 5 \mathrm{eV}$ in the TOF spectrum excited by the HH cutoff. We adopted this ion yield as the indicator of the AC signal for the cutoff $\mathrm{HH}(\sim 30 \mathrm{eV})$, because a higher $\mathrm{KE}$ is expected to contain the signal from Coulomb explosion of $\mathrm{N}_{2}^{2+} \rightarrow \mathrm{N}^{+}+\mathrm{N}^{+}$(ref. 34) via a two-photon double ionization driven by the cutoff $\mathrm{HH}$. As we could also measure the attosecond pulse train structure when the OC-HH cutoff was employed, we can conclude that the side-peak signal at $5 \mathrm{eV}$ originates from a nonlinear interaction of the $\mathrm{HH}$ fields with the $\mathrm{N}_{2}$ molecules. As is shown in Figs 2 and 3, the appearance of the AC signal itself in the TOF mass spectra unambiguously proves that there is a nonlinear interaction of $\mathrm{HH}$ fields resulting in the production of $\mathrm{N}^{+}$ions at each $\mathrm{KE}$, as a linear interaction cannot provide a correlated signal of the pulse envelope.

To discuss the detection sensitivity of our AC measurements, we evaluated the noise-to-peak intensity ratio of the AC trace. Here we defined this ratio according to the following procedure. First, we fit the experimental data points in the middlebottom panel of Fig. 3 to a Gaussian function. We determined the amplitude of the AC peak $\left(V_{\mathrm{amp}}\right)$ by the fit Gaussian function trace. Next, we subtract the fit Gaussian function trace from the experimental data and evaluated the s.d. $\left(V_{\text {sdev }}\right)$ as the background signal. The signal fluctuations are caused by fluctuations of the measured $\mathrm{N}^{+}$ion yield. The noise-to-peak intensity ratio is defined by $V_{\text {sdev }} / V_{\text {amp }}$, resulting in 0.24 . Note that we have also evaluated $V_{\text {sdev }}$ by simply picking up data points in the ranges outside the correlation peak, which are $(-1.15 \mathrm{fs},-0.85 \mathrm{fs})$ and $(0.85 \mathrm{fs}, 1.15 \mathrm{fs})$; again we obtained $V_{\text {sdev }} / V_{\text {amp }}$ to be 0.21 . Thus, we conclude that we can notice side-peak signals with amplitude higher than 0.24 times the main peak height in our AC measurements. Note that the signal-to-background ratio of all our (volume-integrated) second-order AC traces is lower than expected from the theory of the volume-integrated second-order AC. This lower ratio has the same origin as in our past attosecond pulse train measurement, namely the contribution of the single-photon ionization to the background ${ }^{35}$.

\section{References}

1. Hentschel, M. et al. Attosecond metrology. Nature 414, 509-513 (2001).

2. Krausz, F. \& Ivanov, M. Attosecond physics. Rev. Mod. Phys. 81, 163-234 (2009).

3. Schafer, K. J., Yang, B., DiMauro, L. F. \& Kulander, K. C. Above threshold ionization beyond the high harmonic cutoff. Phys. Rev. Lett. 70, 1599-1602 (1993).

4. Corkum, P. B. Plasma perspective on strong-field multiphoton ionization. Phys. Rev. Lett. 71, 1994-1997 (1993).

5. Takahashi, E. J., Nabekawa, Y., Otsuka, T., Obara, M. \& Midorikawa, K. Generation of highly coherent submicrojoule soft $\mathrm{x}$ rays by high-order harmonics. Phys. Rev. A 66, 021802(R) (2002).

6. Takahashi, E. J., Nabekawa, Y. \& Midorikawa, K. Generation of 10- $\mu$ J coherent extreme-ultraviolet light by use of high-order harmonics. Opt. Lett. 27, 1920-1922 (2002).

7. Tzallas, P., Charalambidis, D., Papadogiannis, N. A., Witte, K. \& Tsakiris, G. D. Direct observation of attosecond light bunching. Nature 426, 267-271 (2003).

8. Nabekawa, Y. et al. Interferometric autocorrelation of an attosecond pulse train in the single-cycle regime. Phys. Rev. Lett. 97, 153904 (2006).

9. Gauthier, D. et al. Single-shot femtosecond X-ray holography using extended references. Phys. Rev. Lett. 105, 093901 (2010).

10. Togashi, T. et al. Intense coherent light in extreme ultraviolet region by free electron laser seeded with 13th harmonic of Ti:sapphire laser. Opt. Express 19, 317-324 (2011).

11. Goulielmakis, E. et al. Single-cycle nonlinear optics. Science 320, 1614-1617 (2008).

12. Sansone, G., Poletto, L. \& Nisoli, M. High-energy attosecond light sources. Nat Photonics 5, 655-663 (2011).

13. Ferrari, F. et al. High-energy isolated attosecond pulses generated by abovesaturation few-cycle fields. Nat. Photonics 4, 875-879 (2010).

14. Takahashi, E. J., Lan, P., Mücke, O. D., Nabekawa, Y. \& Midorikawa, K. Infrared two-color multicycle laser field synthesis for generating an intense attosecond pulse. Phys. Rev. Lett. 104, 233901 (2010).

15. Constant, E. et al. Optimizing high harmonic generation in absorbing gases: model and experiment. Phys. Rev. Lett. 82, 1668-1671 (1999).

16. Ayvazyan, V. et al. Generation of GW radiation pulses from a VUV freeelectron laser operating in the femtosecond regime. Phys. Rev. Lett. 88, 104802 (2002).

17. Shintake, T. et al. A compact free-electron laser for generating coherent radiation in the extreme ultraviolet region. Nat. Photonics 2, 555-559 (2008).

18. Lan, P., Takahashi, E. J. \& Midorikawa, K. Optimization of infrared two-color multicycle field synthesis for intense-isolated-attosecond-pulse generation. Phys. Rev. A 82, 053413 (2010).

19. Takahashi, E. J., Hasegawa, H., Nabekawa, Y. \& Midorikawa, K. Highthroughput, high-damage-threshold broadband beam splitter for highorder harmonics in the extreme-ultraviolet region. Opt. Lett. 29, 507-509 (2004).

20. Skantzakis, E., Tzallas, P., Kruse, J., Kalpouzos, C. \& Charalambidis, D. Coherent continuum extreme ultraviolet radiation in the sub-100-nJ range generated by a high-power many-cycle laser field. Opt. Lett. 34, 1732-1734 (2009).

21. Tzallas, P., Skantzakis, E., Nikolopoulos, L. A. A., Tsakiris, G. D. \& Charalambidis, D. Extreme-ultraviolet pump-probe studies of onefemtosecond-scale electron dynamics. Nat. Phys. 7, 781-784 (2011).

22. Takahashi, E. J., Nabekawa, Y. \& Midorikawa, K. Low-divergence coherent soft $\mathrm{x}$-ray source at $13 \mathrm{~nm}$ by high-order harmonics. Appl. Phys. Lett. 84, 4-6 (2004).

23. Sekikawa, T., Kosuge, A., Kanai, T. \& Watanabe, S. Nonlinear optics in the extreme ultraviolet. Nature 432, 605-608 (2004).

24. Singh, K. P. et al. Control of electron localization in deuterium molecular ions using an attosecond pulse train and a many-cycle infrared pulse. Phys. Rev. Lett. 104, 023001 (2010)

25. Schafer, K. J., Gaarde, M. B., Heinrich, A., Biegert, J. \& Keller, U. Strong field quantum path control using attosecond pulse trains. Phys. Rev. Lett. 92, 023003 (2004).

26. Takahashi, E. J., Kanai, T., Ishikawa, K. L., Nabekawa, Y. \& Midorikawa, K. Dramatic enhancement of high-order harmonic generation. Phys. Rev. Lett. 99, 053904 (2007).

27. Tate, J. et al. Scaling of wave-packet dynamics in an intense midinfrared field. Phys. Rev. Lett. 98, 013901 (2007). 
28. Lan, P., Takahashi, E. J. \& Midorikawa, K. Wavelength scaling of efficient highorder harmonic generation by two-color infrared laser fields. Phys. Rev. A 81, 061802 (2010).

29. Takahashi, E. J., Kanai, T., Nabekawa, Y. \& Midorikawa, K. 10-mJ class femtosecond optical parametric amplifier for generating soft $\mathrm{x}$-ray harmonics. Appl. Phys. Lett. 93, 041111 (2008).

30. Krumrey, M., Tegeler, E., Goebel, R. \& Kohler, R. Self-calibration of the same silicon photodiode in the visible and soft x-ray ranges. Rev. Sci. Instrum. 66, 4736-4737 (1995).

31. Powell, F. R., Vedder, P. M., Lindblom, J. F. \& Powell, S. F. Thin film filter performance for extreme ultraviolet and x-ray applications. Opt. Eng. 29, 614-624 (1990).

32. Aoto, T. et al. Inner-valence states of $\mathrm{N}_{2}^{+}$and the dissociation dynamics studied by threshold photoelectron spectroscopy and configuration interaction calculation. J. Chem. Phys. 124, 234306 (2006).

33. Wetmore, R. W. \& Boyd, R. K. Theoretical investigation of the dication of molecular nitrogen. J. Phys. Chem. 90, 5540-5551 (1986).

34. Sato, T. et al. Dissociative two-photon ionization of $\mathrm{N}_{2}$ in extreme ultraviolet by intense self-amplified spontaneous emission free electron laser light. Appl. Phys. Lett. 92, 154103 (2008).

35. Nabekawa, Y. \& Midorikawa, K. Interferometric autocorrelation of an attosecond pulse train calculated using feasible formulae. New J. Phys. 10, 025034 (2008).

36. Sansone, G. et al. Isolated single-cycle attosecond pulses. Science 314, 443-446 (2006).

37. Mashiko, H. et al. Double optical gating of high-order harmonic generation with carrier-envelope phase stabilized lasers. Phys. Rev. Lett. 100, 103906 (2008).

38. Feng, X. et al. Generation of isolated attosecond pulses with 20 to 28 femtosecond lasers. Phys. Rev. Lett. 103, 183901 (2009).

\section{Acknowledgements}

This work was supported by the Ministry of Education, Culture, Sports, Science and Technology (MEXT) through a grant for Extreme Photonics Research; by the Japan Society for the Promotion of Science through a Grant-in-Aid for Scientific Research; by the MEXT through a Grant-in-Aid for Scientific Research for Young Scientists (B) No. 23760057; and through a Grant-in-Aid for Scientific Research (B) No. 25286074. Y.N. gratefully acknowledges the financial support by Grant-in-Aid for Scientific Research (A) No. 21244066.

\section{Author contributions}

E.J.T. was the main contributor in all aspects of this project; he conceived and designed the experiment. He also wrote the manuscript, which was polished by all the authors. E.J.T., P.L. and O.D.M. discussed the generation scheme for obtaining an intense IAP. P.L. performed the theoretical calculations. Y.N. contributed to the AC experiments. K.M. oversaw this project as a whole.

\section{Additional information}

Competing financial interests: The authors declare no competing financial interests

Reprints and permission information is available online at http://www.nature.com/ reprintsandpermissions.

How to cite this article: Takahashi, E. J. et al. Attosecond nonlinear optics using gigawatt-scale isolated attosecond pulses. Nat. Commun. 4:2691 doi: 10.1038/ncomms3691 (2013).

(c) (1) $\odot$ This work is licensed under a Creative Commons Attribution-

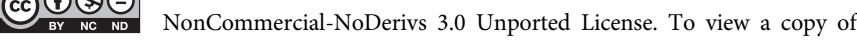
this license, visit http://creativecommons.org/licenses/by-nc-nd/3.0/ 